\title{
Missing-data analysis: socio- demographic, clinical and lifestyle determinants of low response rate on self- reported psychological and nutrition related multi- item instruments in the context of the ATTICA epidemiological study
}

Thomas Tsiampalis ${ }^{1}$ and Demosthenes B. Panagiotakos ${ }^{1,2^{*}}$

\begin{abstract}
Background: Missing data is a common problem in epidemiological studies, while it becomes more critical, when the missing data concern a multi-item instrument, since lack of information in even one of its items, leads to the inability to calculate the total score of the instrument. The aim was to investigate the socio-demographic, lifestyle and clinical determinants of low response rate in two self- rating multi item scales, estimating the individuals' nutritional habits and psychological disorders, as well as, to compare different missing data handling techniques regarding the imputation of missing values in this context.

Methods: The sample from ATTICA epidemiological study was used, with complete baseline information (20012002) regarding their demographic characteristics $[n=2194$ subjects (1364 men: 64 years old (SD $=12$ years) and 830 women: 66 years old $(S D=12$ years))]. Adherence to the Mediterranean diet and depressive symptomatology were assessed at baseline, with the MedDietScore scale and the Zung's Self- rating Depression Scale (SDS), respectively. Logistic and Poisson regression analysis were used, in order to explore the low response's determinants in each scale. Seven missing data handling techniques were compared in terms of the estimated regression coefficients and their standard errors, under different scenarios of missingness, in the context of a multivariable logistic regression model examining the association of each scale with the participants' likelihood of being hypertensive.
\end{abstract}

\footnotetext{
* Correspondence: dbpanag@hua.gr
}

'Department of Nutrition and Dietetics, School of Health Science and Education, Harokopio University, 70 Eleftheriou Venizelou Ave., 17671 Athens, Greece

${ }^{2}$ Faculty of Health, University of Canberra, Canberra, Australia

(c) The Author(s). 2020 Open Access This article is licensed under a Creative Commons Attribution 4.0 International License, which permits use, sharing, adaptation, distribution and reproduction in any medium or format, as long as you give appropriate credit to the original author(s) and the source, provide a link to the Creative Commons licence, and indicate if changes were made. The images or other third party material in this article are included in the article's Creative Commons licence, unless indicated otherwise in a credit line to the material. If material is not included in the article's Creative Commons licence and your intended use is not permitted by statutory regulation or exceeds the permitted use, you will need to obtain permission directly from the copyright holder. To view a copy of this licence, visit http://creativecommons.org/licenses/by/4.0/ The Creative Commons Public Domain Dedication waiver (http://creativecommons.org/publicdomain/zero/1.0/) applies to the data made available in this article, unless otherwise stated in a credit line to the data. 
(Continued from previous page)

Results: Older age, lower educational level, poorer health status and unhealthy lifestyle habits, were found to be significant determinants of high nonresponse rates, both in the MedDietScore scale and the Zung's SDS. Female participants were more likely to have missing data in the items of the MedDietScore scale, while a significantly higher number of missing items in the depression scale was found for male participants. Concerning the analysis of such data, multiple imputation was found to be the most effective technique, even when the number of missing items was large.

Conclusions: The present work augments prior evidence that higher non-response to health surveys is significantly affected by responders' background characteristics, while it gives rise to research towards unrevealed paths behind this claim, especially in the era of nutritional epidemiology.

Keywords: Multi- item scales, Missing data, Imputation, Mediterranean diet, Depression, Low response rate

\section{Background}

Missing data is a common phenomenon, especially, in questionnaire-based, population surveys or epidemiological studies. Presence of missing data reduce the representativeness of the selected sample, cause bias and lead to a decrease in the a-priori designed statistical power, as well as the efficiency and validity of the conducted analyses and therefore, distort inferences about the referent population $[1,2]$. Although several methodological frameworks have been proposed to reduce missingness in data collection in quantitative surveys, this situation is, unfortunately, quite common in research. Moreover, it becomes more critical, especially when the missing data concern a multi-item, health-related instrument (or scale, score), which is applied to measure a latent construct that is difficult or impossible to measured directly [3]. There is a variety of such instruments that has been developed to measure psychological disorders' symptomatology (like anxiety, depression, stress) [4], dietary patterns (like Mediterranean diet) and behaviors (like Healthy Eating pattern) [5], and several clinical conditions (like risk of developing cardiovascular disease (CVD), diabetes, obesity) [6]. Lack of information in even one of the instruments' items, leads to the inability to calculate the total score of the instrument, making the whole procedure useless since it would not be able to correctly classify the individual to the health class belongs.

The main sources of item's non-response are, the type of research (e.g., topic of research, referent population), the structure of the questionnaire or the instrument, the interviewer (e.g., easy acceptance of don't' knows (DKs)), and the background characteristics of the respondents [7-9]. Identifying the profile of individuals with missing data, is of crucial importance in order for a study and its results to be valid. For instance, individuals with missing data may be systematically different from those with complete information, either regarding the outcome of interest, or their prognosis in general. Review of the source of missingness in health surveys revealed that older individuals and low educated, as well as, females and those with poorer health status, tend to have higher levels of missing information [10].

Although several methodologies have been proposed, the aforementioned topic of missing data analysis is still not well studied and understood [11]. Complete case analysis (CCA) and proration (i.e., summing or averaging the available items with no missing data) constitute two of the most frequently used missing data handling methods $[12,13]$. In spite of their simplicity, methodologists have raised several important concerns about their use, since they lead to underpowered results caused by a decreased sample size and they depend on missing data patterns and rates in the sample [14]. According to Rubin's terminology, missing data patterns are classified as missing completely at random (MCAR) where the probability of missingness does not depend on either observed or missing data, missing at random (MAR) where conditional on the observed data, the probability of missingness is independent of unobserved data, and missing not at random (MNAR), where the probability of missingness is dependent on unobserved data even after conditioning on observed data [15].

The aim of the present work was (a) to investigate the demographic, clinical and lifestyle profile of the participants of the ATTICA epidemiological study, with missing data in two health- related scales that aimed to evaluate a psychological condition (depression) and adherence to a dietary pattern (Mediterranean diet), as well as, (b) to investigate the performance of different missing data handling methodologies on the aforementioned instruments and compare them in terms of the level on which they affect both the magnitude of the studied relationship and its uncertainty, as expressed by the standard error.

\section{Methods}

Sample

The working sample to test the research hypothesis of this work is the data from the ATTICA study, which is a prospective, observational cohort investigation initiated 
in 2001 [16]. At the baseline examination (2001-2002), $n=3042$ apparently healthy volunteers (free of CVD and other chronic diseases) residing in the greater metropolitan Athens area, in Greece, agreed to participate (75\% participation rate). Of the enrolled participants, $\mathrm{n}=1514$ $(49.8 \%)$ were men [ 46 years old ( $\mathrm{SD}=13$ years)] and $\mathrm{n}=$ $1528(50.2 \%)$ were women [45 years old ( $\mathrm{SD}=14$ years)]. During baseline examination, a detailed clinical evaluation was performed by trained physicians. For the purposes of this work, we excluded $n=848$ participants with missing or incomplete demographic information and, thus the working sample consisted of $n=2194$ subjects [1364 men: 64 years old ( $\mathrm{SD}=12$ years) and 830 women: 66 years old $(\mathrm{SD}=12$ years $)$ ].

\section{Bioethics}

ATTICA study was approved by the Bioethics Committee of Athens Medical School. The study was carried out in accordance with the Declaration of Helsinki (1989) of the World Medical Association. All participants were informed about the study aims and procedures and provided written informed consent.

\section{Baseline measurements}

\section{Socio-demographic, anthropometric and lifestyle} characteristics

The socio- demographic, anthropometric and lifestyle characteristics assessed, included among others age (in years), sex (male/ female), educational level (No formal studies/ Primary education ( $\leq 6$ years)/ Secondary education ( $\leq 12$ years)/ Higher education $(>12$ years)), body mass index (according to standard guidelines obesity was defined as body mass index > $29.9 \mathrm{Kg} / \mathrm{m}^{2}$ ), as well as, physical activity level (measured in MET/week) and smoking status, based on which participants were classified for the purposes of this work in two groups: Group I: Healthy lifestyle = non- smokers and physically active participants and Group II: Unhealthy lifestyle=Either smokers, or physically inactive participants.

Further details regarding the methods and measurements applied in the ATTICA study have been previously detailed [16].

\section{Clinical characteristics}

Assessment of clinical characteristics (hypertension, hypercholesterolemia, and diabetes mellitus) was performed according to established physical examination procedures and pharmaceutical treatment [16]. In particular, diabetes mellitus was defined as a fasting blood sugar $>125 \mathrm{mg} / \mathrm{dl}$ or the use of antidiabetic medication and, thus, participants were classified as diabetic or non- diabetic. Patients whose average blood pressure levels that were measured by study's investigators through standard procedure, were greater or equal to $140 / 90 \mathrm{mmHg}$ or were under antihypertensive medication, were classified as having hypertension. Based on the total serum cholesterol levels measured, participants were classified in three groups (Group I: Desirable levels (<200 mg/dL), Group II: Borderline levels (200-239 mg/dL) and Group III: High levels $(>240 \mathrm{mg} / \mathrm{dL})$ ), with those belonging in Group II and III, characterized as hypercholesterolemic.

\section{Dietary assessment}

The MedDietScore, an instrument (scale) used to estimate the level of adherence to the Mediterranean diet, was applied to all participants [5]. This scale consists of 11 items estimating the frequency with which individuals consume several foods, which are either close to the Mediterranean diet (e.g., fruits, vegetables, non-refined cereals, and products), or away (e.g. meat and meat products). Higher values of this scale indicate adherence to the traditional Mediterranean diet, while lower values indicate adherence to the "Westernized" diet.

\section{Psychological evaluation}

A translated and validated version of the Zung's SelfRating Depression Scale (SDS) was used, in order to assess the depressive symptoms of the participants. The scale consists of 20 items, covering affective, cognitive, and somatic symptoms, which estimate the frequency with which each symptom is experienced by the individual. Higher scores are indicative of more severe depression [17].

\section{Outcomes}

The outcome examined in the present work was the number of missing data in the items of the two selfrating scales (i.e., MedDietScore and Zung's SDS). Specifically, for each participant two new variables were created indicating the number of missing items in each scale. As far as the MedDietScore is concerned, participants were further classified, as those without missing data and those with missing data in at least one item, in order to investigate the characteristics of those with missing data. Concerning the Zung's SDS, three more variables were created indicating the number of missing items in each subscale estimating the affective, cognitive, and somatic symptoms. Furthermore, in order to examine the behavior of the different missing data handling techniques with an increasing number of missing data (in each scale), participants were further classified in 3 groups, based on the number of missing items in the total MedDietScore and Zung's SDS scale. 


\section{Statistical analysis}

Continuous variables are presented as mean values (standard deviation, SD) and categorical variables are presented as relative frequencies (\%).

\section{Investigation of the participants' profile with missing data}

Associations between categorical variables and the binary (no missing data/missing data in at least one item) form of the number of missing data in the MedDietScore scale, were tested with the Pearson Chi square test. Associations between the number of missing data in each scale or subscale with categorical variables, were tested with the independent samples t- test (in case of 2 categories) and the One-way ANOVA (in case of $\geq 3$ categories). Whether these variables were normally distributed was tested through P-P plot and equality of variances through

Table 1 Distribution of the participants' demographic, clinical and lifestyle characteristics, for the total sample and separately according to the level of missingness in the MedDietScore scale; the ATTICA epidemiological study

\begin{tabular}{|c|c|c|c|c|c|c|}
\hline & $\begin{array}{l}\text { Total sample } \\
(N=2194)\end{array}$ & $\begin{array}{l}\text { No missing } \\
\text { data }(N=775)\end{array}$ & $\begin{array}{l}\text { Missing data in at least } \\
\text { one item of the } \\
\text { MedDietScore } \\
\text { scale }(N=1419)\end{array}$ & $\boldsymbol{p}$-value & OR $(95 \% \mathrm{Cl})^{2}$ & $\boldsymbol{p}$-value \\
\hline \multicolumn{7}{|l|}{ Demographic characteristics } \\
\hline Age [years; Mean $\left.\left(\mathrm{SD}^{4}\right)\right]$ & $64.81(11.86)$ & 64.79 (11.77) & $64.82(11.90)$ & 0.952 & $1.00(0.99,1.01)$ & 0.952 \\
\hline Sex $(\%)$ & & & & 0.913 & & \\
\hline Male & 62.2 & 35.4 & 64.6 & & 1.00 & - \\
\hline Female & 37.8 & 35.2 & 64.8 & & $1.01(0.84,1.21)$ & 0.913 \\
\hline \multicolumn{7}{|l|}{ Educational level $(\%)^{5}$} \\
\hline No formal studies & 3.9 & 19.5 & 80.5 & $<0.001$ & $2.65(1.14,6.12)$ & 0.023 \\
\hline Primary education & 26.6 & 20.6 & 79.4 & & $2.48(1.60,3.83)$ & $<0.001$ \\
\hline Secondary education & 55.3 & 27.3 & 72.7 & & $1.71(1.18,2.48)$ & 0.005 \\
\hline Higher education & 14.2 & 39.1 & 60.9 & & 1.00 & - \\
\hline \multicolumn{7}{|l|}{ Clinical characteristics } \\
\hline \multicolumn{7}{|l|}{ Obesity $(\%)^{6}$} \\
\hline Yes & 26.1 & 35.9 & 64.1 & 0.956 & $1.01(0.82,1,23)$ & 0.956 \\
\hline No & 73.9 & 36.0 & 64.0 & & 1.00 & - \\
\hline \multicolumn{7}{|l|}{ Diabetes Mellitus $(\%)^{7}$} \\
\hline Yes & 31.9 & 36.6 & 63.4 & 0.042 & $1.32(1.01,1.72)$ & 0.042 \\
\hline No & 68.1 & 43.2 & 56.8 & & 1.00 & - \\
\hline \multicolumn{7}{|l|}{ Hypertension $(\%)^{8}$} \\
\hline Yes & 65.6 & 35.4 & 64.6 & 0.900 & $0.99(0.82,1.19)$ & 0.900 \\
\hline No & 34.4 & 35.1 & 64.9 & & 1.00 & - \\
\hline \multicolumn{7}{|l|}{ Hypercholesterolemia $(\%)^{9}$} \\
\hline Desirable levels (< 200 mg/dL) & 33.1 & 47.2 & 52.8 & $<0.001$ & $0.50(0.40,0.62)$ & $<0.001$ \\
\hline Borderline levels (200-239 mg/dL) & 26.4 & 36.0 & 64.0 & & $0.79(0.63,0.98)$ & 0.048 \\
\hline High levels (> 240 mg/dL) & 40.5 & 30.9 & 69.1 & & 1.00 & - \\
\hline \multicolumn{7}{|l|}{ Lifestyle characteristics } \\
\hline \multicolumn{7}{|l|}{ Type of lifestyle $(\%)^{10}$} \\
\hline Unhealthy lifestyle & 83.2 & 15.0 & 85.0 & 0.165 & $1.33(0.89,1.98)$ & 0.165 \\
\hline Healthy lifestyle & 16.8 & 19.0 & 81.0 & & 1.00 & - \\
\hline
\end{tabular}

Notes: ${ }^{1} p$-value is based on the Pearson Chi- square test in case of the categorical characteristics and on the Independent samples t-test in case of the continuous characteristics. ${ }^{2} \mathrm{OR}=$ Odds ratio, $\mathrm{Cl}=$ Confidence Interval. ${ }^{3} \mathrm{p}$-value refers to the comparison of each category with the baseline category, while in the case of participants' age it refers to 1 - year increase. ${ }^{4} \mathrm{SD}=$ Standard Deviation. ${ }^{5}$ Educational level is defined as follows: No formal studies $=0$ years, Primary education $\leq 6$ years, Secondary education $\leq 12$ years, and Higher education $>12$ years. ${ }^{6}$ Obesity was defined as Body Mass $\mathrm{Index}(\mathrm{BMI}) \geq 29.9 \mathrm{~kg} / \mathrm{m}^{2}$. ${ }^{7}$ Diabetes mellitus was defined as a fasting blood sugar $>125 \mathrm{mg} / \mathrm{dl}$ or the use of antidiabetic medication. ${ }^{8}$ Patients whose average blood pressure levels were greater or equal to $140 / 90 \mathrm{mmHg}$ or were under antihypertensive medication were classified as hypertensives. ${ }^{9}$ The definition of hypercholesterolemia was based on the total serum cholesterol levels. ${ }^{10}$ Healthy lifestyle $=$ non- smokers and physically active participants, Unhealthy lifestyle $=$ Either smokers, or physically inactive participants 
Table 2 Average number of missing items in the MedDietScore scale, according to the participants' demographic, clinical and lifestyle characteristics; the ATTICA epidemiological study

\begin{tabular}{|c|c|c|c|c|}
\hline & Mean $\left(\mathrm{SD}^{7}\right)$ & $p$-value ${ }^{2}$ & $\operatorname{IRR}(95 \% \mathrm{CI})^{3}$ & $p$-value \\
\hline \multicolumn{5}{|l|}{ Demographic characteristics } \\
\hline \multicolumn{5}{|l|}{ Sex } \\
\hline Male & $2.07(2.65)$ & 0.390 & 1.00 & - \\
\hline Female & $2.17(2.70)$ & & $1.05(0.99,1.11)$ & 0.114 \\
\hline \multicolumn{5}{|l|}{ Educational level $^{5}$} \\
\hline No formal studies & $2.83(2.60)$ & 0.002 & $1.61(1.29,2.00)$ & $<0.001$ \\
\hline Primary education & $2.41(2.61)$ & & $1.37(1.19,1.58)$ & $<0.001$ \\
\hline Secondary education & $2.69(2.90)$ & & $1.53(1.34,1.74)$ & $<0.001$ \\
\hline Higher education & $1.76(2.41)$ & & 1.00 & - \\
\hline \multicolumn{5}{|l|}{ Clinical characteristics } \\
\hline \multicolumn{5}{|l|}{ Obesity $^{6}$} \\
\hline Yes & $2.05(2.63)$ & 0.507 & $1.04(0.97,1.12)$ & 0.222 \\
\hline No & $1.97(2.58)$ & & 1.00 & - \\
\hline \multicolumn{5}{|l|}{ Diabetes Mellitus ${ }^{7}$} \\
\hline Yes & $1.72(2.35)$ & 0.007 & $1.31(1.18,1.45)$ & $<0.001$ \\
\hline No & $1.32(2.05)$ & & 1.00 & - \\
\hline \multicolumn{5}{|l|}{ Hypertension $^{8}$} \\
\hline Yes & $2.07(2.64)$ & 0.448 & $0.96(0.90,1.02)$ & 0.163 \\
\hline No & $2.16(2.71)$ & & 1.00 & - \\
\hline \multicolumn{5}{|l|}{ Hypercholesterolemia $^{9}$} \\
\hline Desirable levels (< 200 mg/dL) & $1.35(2.18)$ & $<0.001$ & $0.63(0.58,0.69)$ & $<0.001$ \\
\hline Borderline levels (200-239 mg/dL) & $1.89(2.52)$ & & $0.89(0.82,0.96)$ & 0.002 \\
\hline High levels (> 240 mg/dL) & $2.13(2.57)$ & & 1.00 & - \\
\hline \multicolumn{5}{|l|}{ Lifestyle characteristics } \\
\hline \multicolumn{5}{|l|}{ Type of lifestyle ${ }^{10}$} \\
\hline Unhealthy lifestyle & $2.86(2.78)$ & 0.022 & $1.20(1.09,1.33)$ & $<0.001$ \\
\hline Healthy lifestyle & $2.38(2.63)$ & & 1.00 & - \\
\hline
\end{tabular}

Notes: $1 \mathrm{SD}=$ Standard Deviation. $2 \mathrm{p}$-value is based on the Independent samples t-test when the categorical characteristic has two categories, and on the oneway Analysis of Variance (ANOVA) when the categorical characteristic has at least three categories. 3 IRR= Incidence Rate Ratio, Cl= Confidence Interval. 4 p-value refers to the comparison of each category to the baseline category. 5 Educational level is defined as follows: No formal studies $=0$ years, Primary education $\leq 6$ years, Secondary education $\leq 12$ years, and Higher education $>12$ years. 6Obesity was defined as Body Mass Index (BMI) $\geq 30 \mathrm{~kg} / \mathrm{m} 2$. 7 Diabetes mellitus was defined as a fasting blood sugar $>125 \mathrm{mg} / \mathrm{dl}$ or the use of antidiabetic medication. 8 Patients whose average blood pressure levels were greater or equal to $140 /$ $90 \mathrm{~mm} \mathrm{Hg}$ or were under antihypertensive medication were classified as hypertensives. 9 The definition of hypercholesterolemia was based on the total serum cholesterol levels. 10 Healthy lifestyle= non- smokers and physically active participants, Unhealthy lifestyle= Either smokers, or physically inactive participants

Levene's test. Odds ratios (OR) and their corresponding 95\% Confidence Intervals $(95 \%$ CI) were evaluated through univariable and multivariable logistic regression analysis, which was used to find the participants' characteristics being significantly associated with the likelihood of having missing data in at least one item of the MedDietScore scale. Incidence Rate Ratios (IRR) and their corresponding 95\% CI were evaluated through univariable and multivariable Poisson regression, which was used to investigate the significant predictors of the average number of missing data in the examined scales and subscales. Backward model selection was used to determine the final significant predictors.

\section{Comparison of different missing data handling techniques}

Seven missing data handling methods were applied here and compared: (1) Complete case analysis (CCA), which leads to biased estimates especially when the data are MNAR, (2) Proration, which results in bias even under a MCAR mechanism, (3) Score mean imputation (SMI), (4) Item mean imputation (IMI), (5) Person mean imputation (PMI), all of which tend to cause biased estimates under every missing data mechanism (MCAR, MAR and MNAR) as the proportion of missing data increases, (6) Stochastic Regression imputation (SRI), which can yield unbiased estimates under the MAR mechanism and (7) Multiple imputation (MI), which 
Obesity VS non- Obesity

No formal studies VS Higher education

Primary education VS Higher education

Secondary education VS Higher education

Borderline levels VS Desirable levels of total serum cholesterol

Higher levels VS Desirable levels of total serum cholesterol

Unhealthy VS Healthy lifestyle

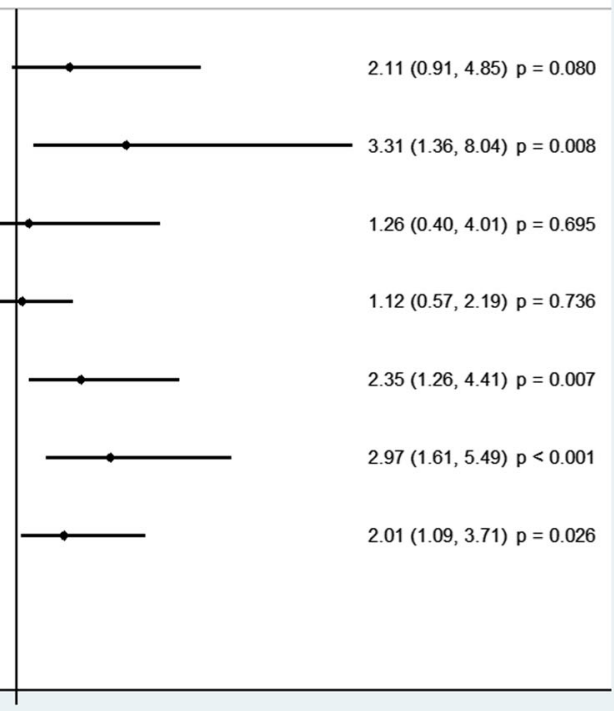

Fig. 1 Statistically significant socio-demographic, clinical and lifestyle determinants of the participants' likelihood of having missing data in at least one item of the MedDietScore scale; the ATTICA epidemiological study. Notes: Results are based on the logistic regression analysis. OR= Odds Ratio, $\mathrm{Cl}=$ Confidence Interval. $\mathrm{p}=\mathrm{p}$-value. Educational level is defined as follows: No formal studies $=0$ years, Primary education $\leq 6$ years, Secondary education $\leq 12$ years and Higher education $>12$ years. Obesity was defined as Body Mass $I n d e x(B M I) \geq 30 \mathrm{~kg} / \mathrm{m}^{2}$. The definition of hypercholesterolemia was based on the total serum cholesterol levels. Healthy lifestyle = non- smokers and physically active participants,

Unhealthy lifestyle $=$ Either smokers, or physically inactive participants

assumes that data are MAR. After applying each method, a multivariable logistic regression model (including participants' age and sex) was fitted, examining the association of each scale with the participants' likelihood of being hypertensive. The aforementioned techniques were compared in terms of the estimated regression coefficients and their standard errors, while they were fitted both in the original dataset as well as, in several subsets defined by the number of missing items in the examined scales.

CCA Only the subjects with complete observations for the two scales were included in the analysis, while all subjects with missing item scores were removed from the data and the model was fitted to the remaining sample.

Proration Prorated scale scores were calculated for each participant, by summing the items without missing data.

SMI The missing scores were imputed with the mean total score of all observed subjects.
IMI A missing item score was imputed with the mean score for all complete data on that item.

PMI The mean score of the items per subject was calculated, and for each subject missing item scores were imputed with this 'personal mean score'.

SRI The missing values in the total scale scores, were imputed with the regression estimates from the observed variables augmented with a normally distributed random error with a variance equal to the variance of the regression model. The regression model included as covariates the participants' characteristics which were found to be significantly associated with the number of missing data in the two scales. In case of the Zung's SDS the variable of hypertension was not used, since it was used as the outcome in the multivariable model for the comparison of the missing data handling techniques.

MI MI was applied to the total scale scores and the imputed values were estimated from the observed variables in the dataset by an imputation algorithm and a random 


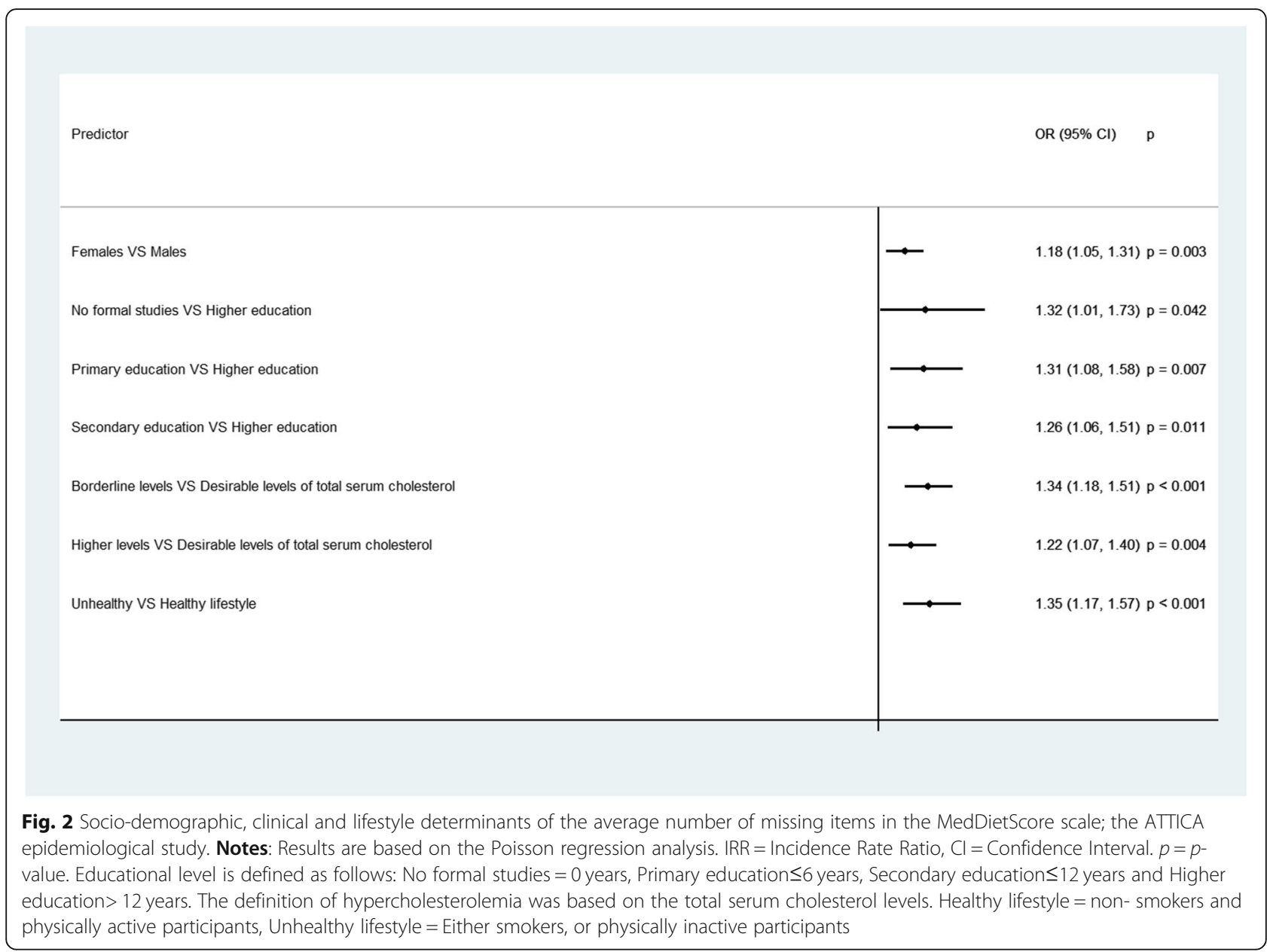

residual term which was added to each resulting estimate. More specifically, the imputation algorithm used was the predictive mean matching, which is appropriate for numeric data, and the imputation model included the participants' characteristics, which were found to be significantly associated with the number of missing data in each scale. In case of the Zung SDS the variable of hypertension was not used, since it was used as the outcome in the multivariable model for the comparison of the missing data handling techniques. Finally, 5 imputed data sets were generated, which is the minimum recommended [18].

All statistical analyses were performed in the STATA software, version 14 [19], except for the SRI and MI, which were performed in $\mathrm{R}$ with the mice package [20].

\section{Results}

\section{Sample characteristics}

The mean age of the participants in the current working sample was 65 years $(\mathrm{SD}=11.86$ years), the majority of them were males (62.2\%) and almost 7 out of $10(69.5 \%)$ were at least in the secondary educational level. The prevalence of the clinical conditions studied were: $26.1 \%$ (obesity), 31.9\% (diabetes), 65.6\% (hypertension) and 66.9\% (hypercholesterolemia), while at least 8 out of 10 participants (83.2\%) were either smokers or physically inactive (unhealthy lifestyle) (Table 1).

\section{Participants' profile with missing data MedDietScore scale}

Participants' with missing data in at least one item of the MedDietScore scale, were less educated, more likely to be diabetic and with higher levels of total serum cholesterol, while participants following an unhealthy lifestyle seemed to have a significantly higher number of missing items in the MedDietScore scale (Tables 1,2). Based on the results from the multivariable models, participants with missing data were more likely to be obese, to have an unhealthy lifestyle, to be less educated, with higher levels of total serum cholesterol, while female participants were also found to have a significantly higher 
Table 3 Average number of missing items in the total Zung Depression scale and in its sub dimensions, for the total sample and according to the participants' demographic, clinical and lifestyle characteristics; the ATTICA epidemiological study

\begin{tabular}{|c|c|c|c|c|c|c|c|c|}
\hline & \multicolumn{2}{|l|}{ Total } & \multicolumn{2}{|c|}{ Affective Symptoms } & \multicolumn{2}{|c|}{ Cognitive Symptoms } & \multicolumn{2}{|c|}{ Somatic symptoms } \\
\hline & Mean $\left(\mathrm{SD}^{\prime}\right)$ & $p$-value ${ }^{2}$ & Mean (SD) & $p$-value ${ }^{2}$ & Mean (SD) & $p$-value ${ }^{2}$ & Mean (SD) & $p$-value \\
\hline Total sample & $7.97(3.74)$ & & $3.28(1.63)$ & & $1.85(1.28)$ & & $0.46(0.97)$ & \\
\hline \multicolumn{9}{|l|}{ Demographic characteristics } \\
\hline \multicolumn{9}{|l|}{ Sex } \\
\hline Male & $8.05(3.71)$ & 0.245 & $3.28(1.62)$ & 0.794 & $1.89(1.27)$ & 0.134 & $0.48(0.97)$ & 0.281 \\
\hline Female & $7.86(3.80)$ & & $3.27(1.64)$ & & $1.80(1.30)$ & & $0.43(0.97)$ & \\
\hline \multicolumn{9}{|l|}{ Educational level $^{3}$} \\
\hline No formal studies & $7.91(3.69)$ & 0.351 & $3.32(1.64)$ & 0.069 & $1.85(1.27)$ & 0.112 & $0.57(1.03)$ & 0.089 \\
\hline Primary education & $7.52(3.74)$ & & $3.15(1.68)$ & & $1.68(1.27)$ & & $0.41(0.91)$ & \\
\hline Secondary education & $7.51(3.94)$ & & $3.13(1.61)$ & & $1.68(1.32)$ & & $0.39(0.89)$ & \\
\hline Higher education & $7.29(3.73)$ & & $2.96(1.59)$ & & $1.54(1.40)$ & & $0.22(0.69)$ & \\
\hline \multicolumn{9}{|l|}{ Clinical characteristics } \\
\hline \multicolumn{9}{|l|}{ Obesity $^{4}$} \\
\hline Yes & $8.06(3.87)$ & 0.515 & $3.28(1.63)$ & 0.870 & $1.86(1.30)$ & 0.840 & $0.52(1.05)$ & 0.113 \\
\hline No & $7.94(3.72)$ & & $3.26(1.62)$ & & $1.85(1.28)$ & & $0.44(0.94)$ & \\
\hline \multicolumn{9}{|l|}{ Diabetes Mellitus ${ }^{5}$} \\
\hline Yes & $8.33(3.52)$ & 0.004 & $3.47(1.59)$ & 0.002 & $1.97(1.24)$ & 0.009 & $0.43(0.91)$ & 0.564 \\
\hline No & $7.64(3.71)$ & & $3.15(1.63)$ & & $1.75(1.30)$ & & $0.39(0.84)$ & \\
\hline \multicolumn{9}{|l|}{ Hypertension $^{6}$} \\
\hline Yes & $8.10(3.74)$ & 0.253 & $3.28(1.63)$ & 0.943 & $1.91(1.28)$ & 0.172 & $0.48(0.98)$ & 0.451 \\
\hline No & $7.91(3.74)$ & & $3.27(1.62)$ & & $1.83(1.28)$ & & $0.45(0.96)$ & \\
\hline \multicolumn{9}{|l|}{ Hypercholesterolemia } \\
\hline Desirable levels $(<200$ mg/dL) & $7.74(3.82)$ & 0.191 & $3.10(1.62)$ & 0.001 & $1.77(1.28)$ & 0.018 & $0.37(0.88)$ & $<0.001$ \\
\hline Borderline levels (200-239 mg/dL) & $7.93(3.82)$ & & $3.19(1.63)$ & & $1.78(1.31)$ & & $0.45(0.95)$ & \\
\hline High levels (> 240 mg/dL) & $8.12(3.65)$ & & $3.42(1.60)$ & & $1.94(1.27)$ & & $0.62(1.10)$ & \\
\hline \multicolumn{9}{|l|}{ Lifestyle characteristics } \\
\hline \multicolumn{9}{|l|}{ Type of lifestyle $^{8}$} \\
\hline Unhealthy lifestyle & $7.73(3.67)$ & 0.821 & $3.23(1.63)$ & 0.518 & $1.79(1.29)$ & 0.855 & $0.44(0.93)$ & 0.351 \\
\hline Healthy lifestyle & $7.66(3.77)$ & & $3.15(1.63)$ & & 1.78 (1.29) & & $0.33(0.88)$ & \\
\hline
\end{tabular}

Notes: ${ }^{1}$ SD Standard Deviation. ${ }^{2} p$-value is based on the Independent samples t-test when the categorical characteristic has two categories, and on the one-way Analysis of Variance (ANOVA) when the categorical characteristic has at least three categories. ${ }^{3}$ Educational level is defined as follows: No formal studies $=0$ years, Primary education $\leq 6$ years, Secondary education $\leq 12$ years, and Higher education $>12$ years. ${ }^{4}$ Obesity was defined as Body Mass $\mathrm{Index}(\mathrm{BMI}) \geq 29.9 \mathrm{~kg} / \mathrm{m}^{2} .{ }^{5}$ Diabetes mellitus was defined as a fasting blood sugar $>125 \mathrm{mg} / \mathrm{dl}$ or the use of antidiabetic medication. ${ }^{6}$ Patients whose average blood pressure levels were greater or equal to $140 / 90 \mathrm{mmHg}$ or were under antihypertensive medication were classified as hypertensives. ${ }^{7}$ The definition of hypercholesterolemia was based on the total serum cholesterol levels. ${ }^{8}$ Healthy lifestyle $=$ non- smokers and physically active participants, Unhealthy lifestyle $=$ Either smokers, or physically inactive participants

number of missing items, when compared to males (Figs. 1, 2).

\section{Zung's self- rating depression scale}

On average, participants did not respond to 8 out of 20 questions of the total instrument, with those indicating the affective symptoms having the lowest response rate, followed by the questions estimating the cognitive symptoms (Table 3). Diabetic participants had a significantly higher nonresponse rate in the total Zung's SDS, while higher levels of total serum cholesterol were significantly associated with higher number of missing items in the three subscales. Based on the results from the multivariable Poisson regression (Fig. 3), men and older participants, those with unhealthy lifestyle, as well as, hypertensive and hypercholesterolemic participants had a significantly higher number of missing items in the total instrument, while diabetic participants had a significantly higher number of missing items in the subscale of cognitive symptoms. 


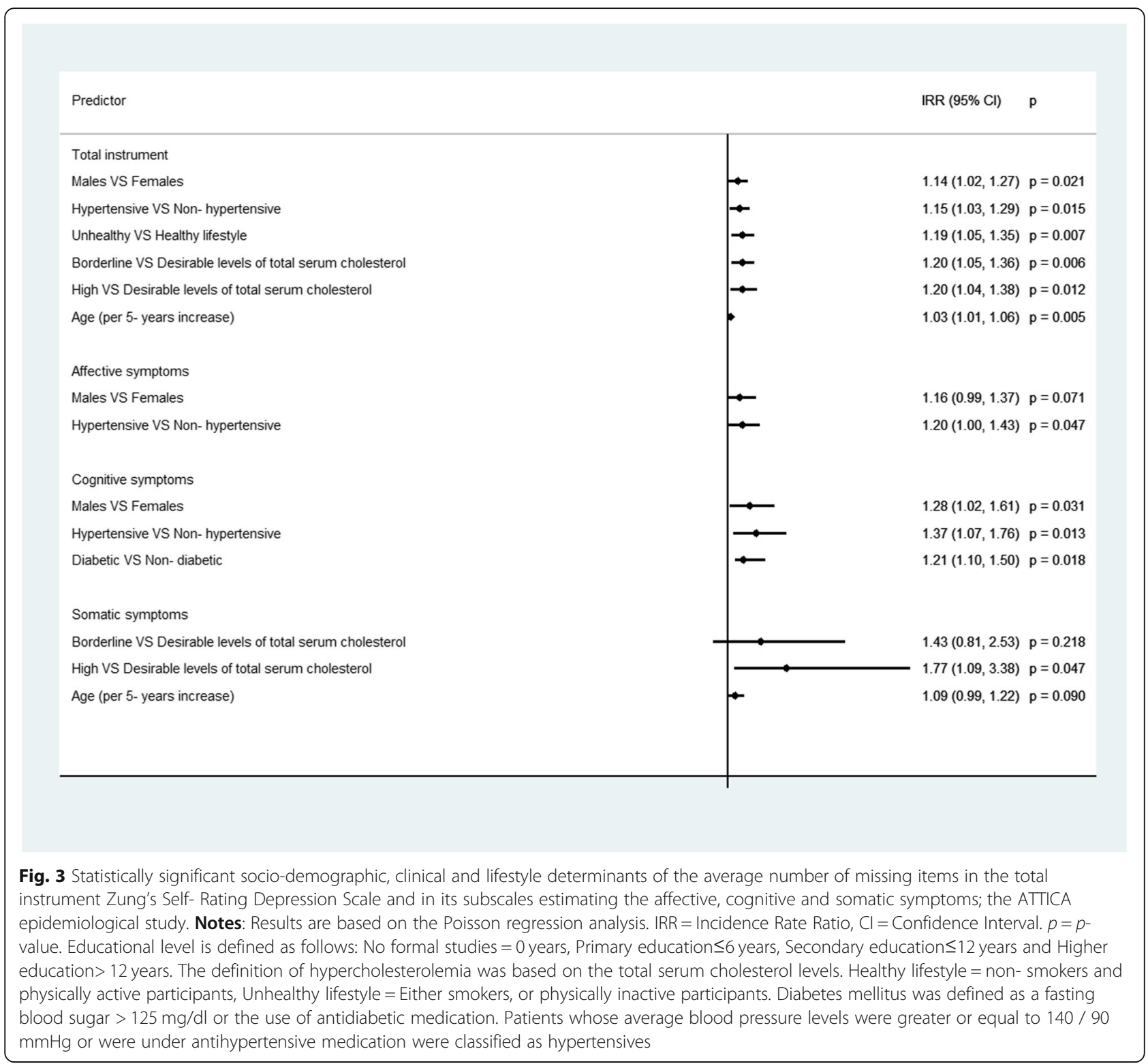

\section{Comparison of missing data handling techniques MedDietScore scale}

In Fig. 4, the beta- coefficient with its standard error is presented, with regard to the effect of the MedDietScore variable on the likelihood of hypertension, after adjusting for participants' age and sex. In all three cases (original data set, missing data in $9-18 \%$ of the items and missing data in $>27 \%$ of the items), the beta- coefficient of the MedDietScore variable did not differ significantly, after applying each missing data handling technique. However, after applying the multiple imputation, the standard error of the coefficient was the lowest, while after applying the stochastic regression imputation, the standard error was the highest. In the original dataset, complete case analysis led to the highest standard error and multiple imputation to the lowest.

\section{Zung's self- rating depression scale}

Multiple imputation led to the lowest standard error, as in the previous case, when participants have missing data either in $5-35 \%$ of the items, or in $>40 \%$ of items, while in the original data set, the coefficients' standard error was the lowest after applying the person mean imputation. As far as the beta coefficient of the Zung's SDS variable is concerned, after applying the stochastic regression imputation, it was significantly higher when compared to the rest missing data handling techniques (Fig. 5). 


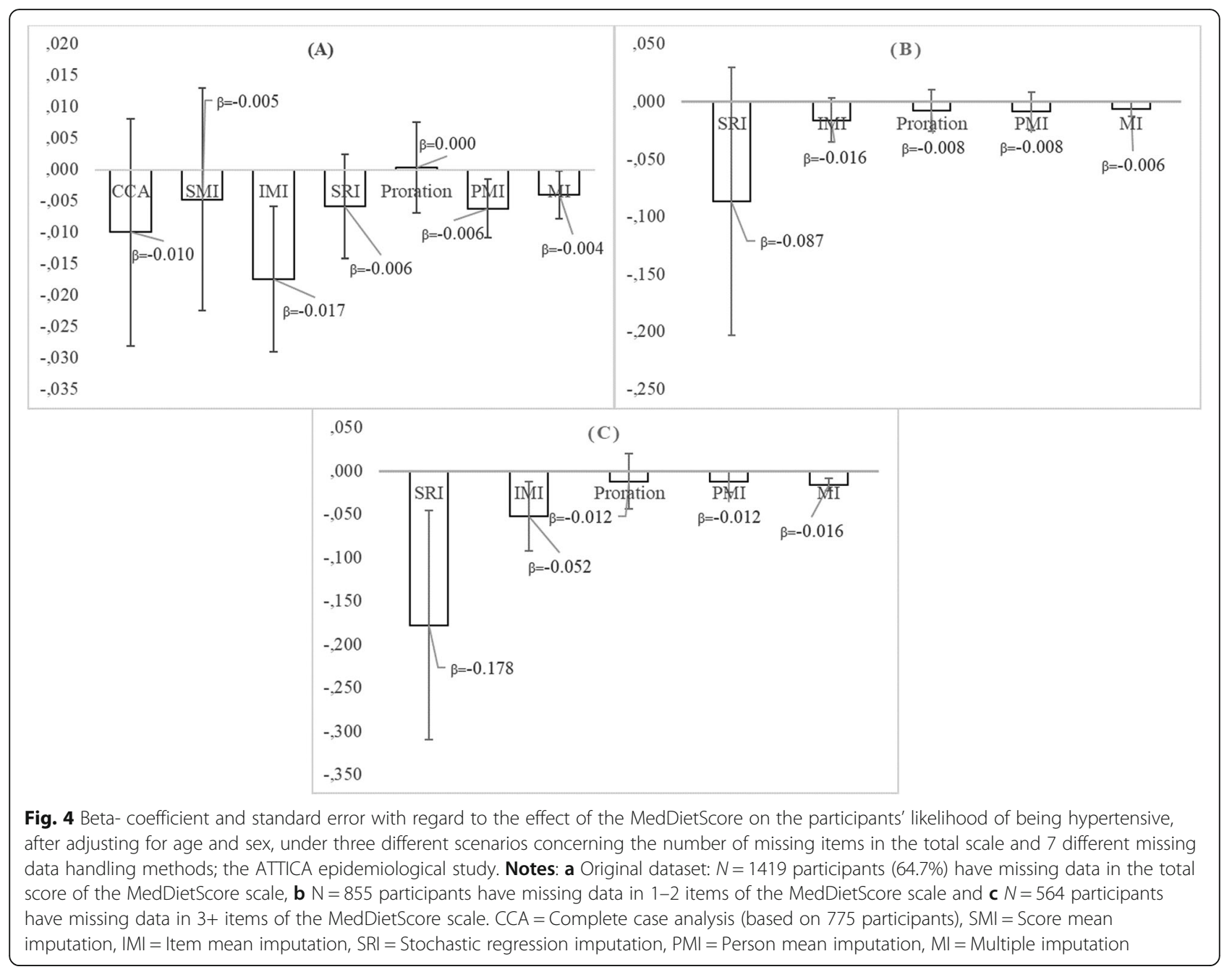

\section{Discussion}

The present work aimed to identify the profile of the individuals with missing data in two multi- item instruments, which are widely used to estimate individuals' adherence to a healthy nutritional pattern and psychological disorders' symptomatology, as well as, to compare some of the most widely used missing data handling techniques with regard to the efficiency and validity of the inferences. Data analyses revealed that the amount of missing data in such structured questionnaires was significantly associated with various demographic, clinical and lifestyle characteristics. In general, higher non- response rate was found to be significantly associated with older age, lower educational level, poorer health status and unhealthy lifestyle in both instruments. In addition, higher number of missing items were reported for female participants in the nutrition- related scale (MedDietScore), while the average number of missing items in the depression scale was significantly higher for male participants. Furthermore, concerning the analysis of such data, removing individuals with missing items seemed to be the worst approach, while multiple imputation was found to be the most effective method, even when the number of missing items was large. Despite the limitations of the present cross- sectional analysis, our findings revealed the profile of participants to whom special focus should be given by the researchers when collecting data, as well as, the importance of imputing the missing values in such cases.

\section{Participants' profile with missing data in the MedDietScore scale}

There is a substantial body of literature investigating the characteristics of the individuals with missing data in surveys, however only a small part of the research focuses on the characteristics of missing data in the context of nutritional epidemiologic studies. Our results seem to agree with those reported by Caan et al. [21], 


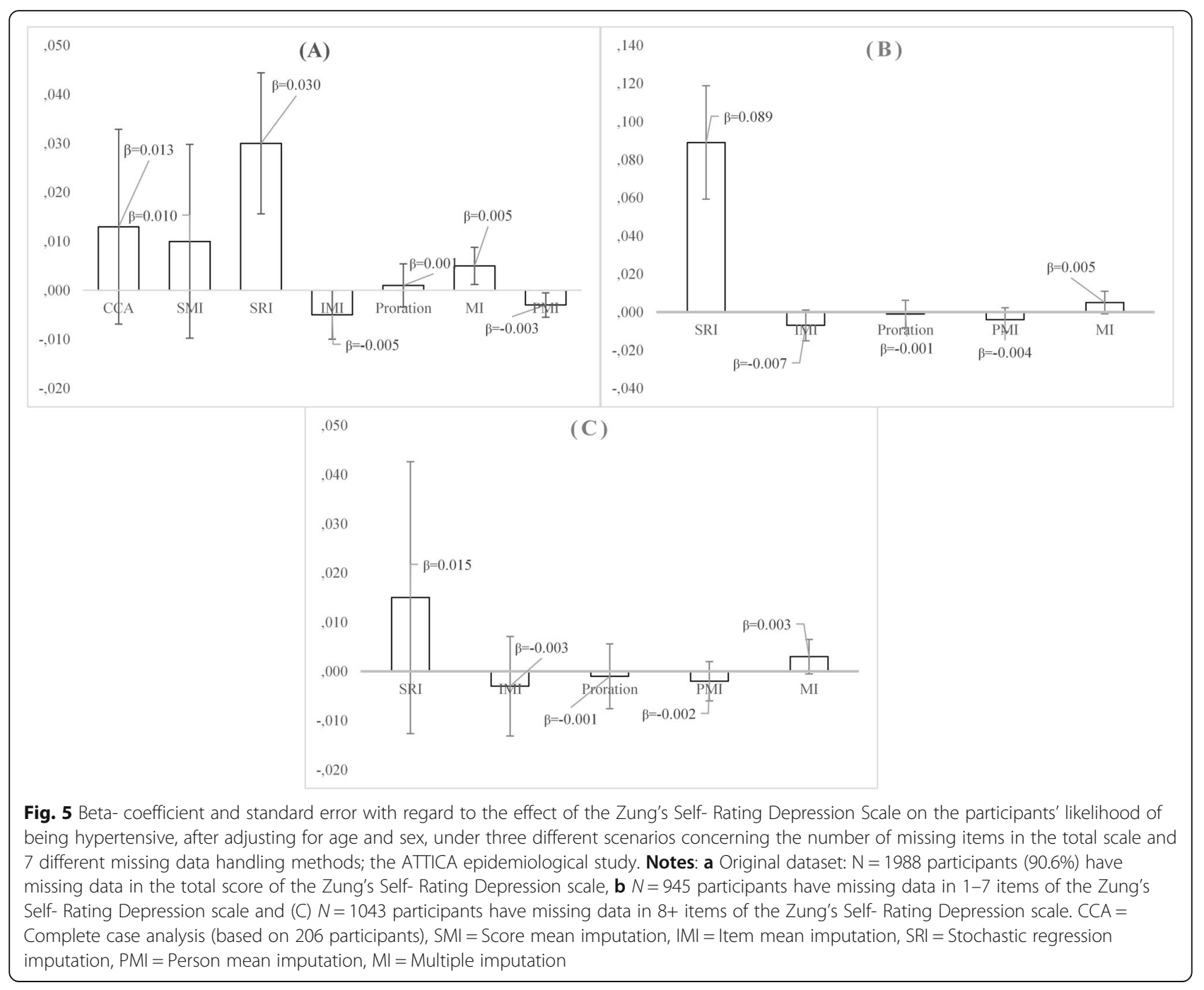

where it was found that less than 3 out of 10 participants responded to the entire questionnaire correctly, while older participants were less likely to respond correctly to the entire survey, which could be attributed to their greater susceptibility to fatigue leading them to skip food items that they do not consume. In addition, the present results are in accordance with another study, where it was reported that several lifestyle factors including age, body mass index, physical activity, and parity are significantly associated with the number of items left blank in a food frequency questionnaires (FFQ), while it was also stated that if more than 20 items on the FFQ are missing, the absolute nutrient intake may be underestimated by more than $10 \%$ [22]. Furthermore, lower educational level, as a proxy measure of the participants' socioeconomic status, was significantly associated with higher non- response in the MedDietScore scale. This result agrees with the study of Wilks et al., who were driven to the same conclusion in the context of a health survey, reporting that individuals in lower socioeconomic groups tend to present higher non- response rates in health surveys [23].

\section{Participants' profile with missing data in the Zung's SDS scale}

The present findings seem to agree with the study conducted by Ying, who found that younger and higher educated men were more likely to respond to the entire instrument (Center for epidemiological studies-depression scale), while middle-aged men and older women were found to have the highest non- response rates [24]. According to Mody et al., older individuals are in a greater risk of item nonresponse by missing or skipping items, either due to cognitive impairment, or due to physical problems, such as vision impairments [25]. In addition, our finding with regard to the lower number of missing items among females, is in accordance with various previous studies 
reporting that female participants are more likely to participate in surveys [26-28]. Moreover, participants' poorer health status was also connected with a higher number of missing items in the Zung's SDS scale, which is in accordance with other studies reporting higher nonresponse rates in individuals with lower subjective health and poorer physical, cognitive, and psychological functioning $[29,30]$.

\section{Missing data handling techniques}

Multiple imputation was found to be the most effective missing data handling technique in terms of the estimated standard error, either compared to the complete case analysis, or to the rest examined methods. Its efficiency over the complete case analysis could be attributed to the fact that MI uses information in the incomplete cases, to the fact that CCA is valid only in the case of MCAR data [31, 32]. Multiple imputation is a general approach which is simple to understand, but hard to program. In addition, yields unbiased estimates and provides more validity, when compared to ad-hoc approaches. Furthermore, multiple imputation was more efficient when compared to mean imputation (at the score or the person or the item level), which is a tempting but not recommended method, as it underestimates the variance in the dataset [29]. In general, our results agree with several other empirical studies in the era of nutritional epidemiology, suggesting that more advanced imputation methods, such as the MI, should be used as they give more accurate intake estimates [33-36].

\section{Limitations}

To the best of our knowledge, this is one of the first studies investigating the profile of individuals with missing data, in such widely used instruments and to such extent. However, the conclusions of the present work should be considered under some existing limitations. First of all, the cross-sectional nature of the data does not allow for causal associations to be drawn. Another limitation is the fact that the true underlying value and the true regression coefficients of the missing data were unknown, as we did not start with a complete data set, which is a usual method in simulation studies comparing different missing data handling methods. Thus, the lack of a simulation study, that would empower the empirical data analyses, could also be considered as a methodological limitation, but this was not the purpose of the present work. Since, in the context of the present study only relative comparisons could be held among the different missing data handling techniques, we cannot conclude which imputation method is more accurate, only that the choice of method may affect both the beta coefficient of the studied relationship, as well as, its standard error. Therefore, our next step in the evaluation of the imputation methods would be to do a simulation study with a complete data set as the reference.

\section{Conclusions}

In summary, older and less educated individuals, as well as, those with morbidities and unhealthier lifestyle habits, constitute a risk group for higher non-response rates when collecting nutrition and psychological data, and therefore, researchers should give special focus when interviewing them, in order to keep the gathered information response rate in high levels. In addition, the results from the applied data analyses revealed that the data imputation methodologies used to complete missing information, preferably the multiple imputation techniques, are trustable and may increase the validity and efficiency of the results.

\section{Abbreviations}

CVD: Cardiovascular Disease; CCA: Complete Case Analysis; MCAR: Missing Completely At Random; MAR: Missing At Random; MNAR: Missing Not At Random; SDS: Self-rating Depression Scale; SD: Standard Deviation;

$\mathrm{Cl}$ : Confidence Interval; OR: Odds Ratio; IRR: Incidence Rate Ratio; SMI: Score Mean Imputation; IMI: Item Mean Imputation; PMI: Person Mean Imputation; SRI: Stochastic Regression Imputation; MI: Multiple Imputation

\section{Acknowledgments}

The authors would like to thank the ATTICA study group of investigators: Christos Pitsavos, Christina Chrysohoou, Yannis Skoumas, Natasa Katinioti, Labros Papadimitriou, Constantina Masoura, Spiros Vellas, Yannis Lentzas, Manolis Kambaxis, Konstanitna Paliou, Vassiliki Metaxa, Agathi Ntzouvani, Dimitris Mpougatsas, Nikolaos Skourlis, Christina Papanikolaou, Aikaterini Kalogeropoulou, Evangelia Pitaraki, Alexandros Laskaris, Mihail Hatzigeorgiou, Athanasios Grekas, and Eleni Kokkou for assistance in the initial physical examination and/or the follow-up evaluation; Efi Tsetsekou for her assistance in psychological evaluation and follow-up evaluation, as well as the laboratory team: Carmen Vassiliadou and George Dedousis (genetic analysis), Marina Toutouza-Giotsa, Constantina Tselika and Sia Poulopoulou (biochemical analysis), and Maria Toutouza for the database management. We would also like to thank all the participants of the ATTICA study.

\section{Authors' contributions}

Conceptualization: T.T. and D.P.; Methodology: T.T. and D.P.; Formal analysis: T.T.; Writing- original draft preparation: T.T.; Writing- review and editing: D.P.; Visualization: T.T.; Supervision: D.P.; Project administration: D.P. Both authors have read and agreed to the published version of the manuscript.

\section{Funding}

The ATTICA study is supported by research grants from the Hellenic Cardiology Society (HCS2002) and the Hellenic Atherosclerosis Society (HAS2003). This work was supported by the Ageing Trajectories of Health: Longitudinal Opportunities and Synergies (ATHLOS) project, which has received funding from the European Union's Horizon 2020 research and innovation program under grant agreement No 635316.

\section{Availability of data and materials}

The data are available upon request. For expression of interest, please contact Prof. Demosthenes Panagiotakos (dbpanag@hua.gr).

\section{Ethics approval and consent to participate}

ATTICA study was approved by the Bioethics Committee of Athens Medical School. The study was carried out in accordance with the Declaration of Helsinki (1989) of the World Medical Association. All participants were 
informed about the study aims and procedures and provided written informed consent.

\section{Consent for publication}

Not applicable.

\section{Competing interests}

Prof. Demosthenes Panagiotakos is a member of the editorial board of BMC Medical Research Methodology. Thomas Tsiampalis declares no conflicts of interest related to this research work.

Received: 2 March 2020 Accepted: 28 May 2020

Published online: 08 June 2020

\section{References}

1. Bell ML, Kenward MG, Fairclough DL, Horton NJ. Differential dropout and bias in randomised controlled trials: when it matters and when it may not. Bmj. 2013;346:e8668

2. Little RJ, Rubin DB. The analysis of social science data with missing values. Sociol Methods Res. 1989;18(2-3):292-326.

3. Panagiotakos D. Health measurement scales: methodological issues. Open Cardiovasc Med J. 2009:3:160.

4. Meites K, Lovallo W, Pishkin V. A comparison of four scales for anxiety, depression, and neuroticism. J Clin Psychol. 1980;36(2):427-32.

5. Panagiotakos DB, Pitsavos C, Stefanadis C. Dietary patterns: a Mediterranean diet score and its relation to clinical and biological markers of cardiovascular disease risk. Nutr Metab Cardiovasc Dis. 2006;16(8):559-68.

6. Kannel WB, McGee D, Gordon T. A general cardiovascular risk profile: the Framingham study. Am J Cardiol. 1976;38(1):46-51.

7. De Leeuw ED, Hox JJ, Huisman M. Prevention and treatment of item nonresponse. J Off Stat. 2003;19:153-76.

8. West BT, Blom AG. Explaining interviewer effects: a research synthesis. J Survey Stat Methodol. 2017;5(2):175-211.

9. Mathiowetz NA. Respondent uncertainty as indicator of response quality. Int J Public Opinion Res. 1999;11(3):289-96.

10. Guadagnoli E, Cleary PD. Age-related item nonresponse in surveys of recently discharged patients. J Gerontol. 1992;47(3):P206-12.

11. Plumpton CO, Morris T, Hughes DA, White IR. Multiple imputation of multiple multi-item scales when a full imputation model is infeasible. BMC Res Notes. 2016;9(1):45

12. Eekhout I, de Boer RM, Twisk JW, de Vet HC, Heymans MW. Missing data: a systematic review of how they are reported and handled. Epidemiology. 2012;23(5):729-32.

13. Schafer $\mathrm{J}$, Graham JW. Missing data: our view of the state of the art. Psychol Methods. 2002;7(2):147.

14. Downey RG, King CV. Missing data in Likert ratings: a comparison of replacement methods. J Gen Psychol. 1998;125(2):175-91.

15. Rubin DB. Inference and missing data. Biometrika. 1976;63(3):581-92.

16. Pitsavos C, Panagiotakos DB, Chrysohoou C, Stefanadis C. Epidemiology of cardiovascular risk factors in Greece: aims, design and baseline characteristics of the ATTICA study. BMC Public Health. 2003;3(1):32.

17. Zung WW. A self-rating depression scale. Arch Gen Psychiatry. 1965;12(1): 63-70.

18. Van Buuren, S. Flexible imputation of missing data. Chapman and Hall/CRC, 2018.

19. StataCorp. 2015. Stata statistical software: release 14. College Station, TX: StataCorp LP.

20. Buuren, S. V., \& Groothuis-Oudshoorn, K. mice: Multivariate imputation by chained equations in R. J Stat Softw, 2010; 1-68.

21. Caan B, Hiatt RA, Owen AM. Mailed dietary surveys: response rates, error rates, and the effect of omitted food items on nutrient values. Epidemiology. 1991:430-6.

22. Michels KB, Willett WC. Self-administered semiquantitative food frequency questionnaires: patterns, predictors, and interpretation of omitted items. Epidemiology (Cambridge, Mass.). 2009;20(2):295.

23. Wilks R, Younger N, Mullings J, Zohoori N, Figueroa P, Tulloch-Reid M, Ward E. Factors affecting study efficiency and item non-response in health surveys in developing countries: the Jamaica national healthy lifestyle survey. BMC Med Res Methodol. 2007;7(1):13.

24. Ying YW. Nonresponse on the center for epidemiological studies-depression scale in Chinese Americans. Int J Soc Psychiatry. 1989;35(2):156-63.
25. Mody L, Miller DK, McGloin JM, Freeman M, Marcantonio ER, Magaziner J, Studenski S. Recruitment and retention of older adults in aging research: (see editorial comments by Dr. Stephanie Studenski, pp 2351-2352). J Am Geriatr Soc. 2008;56(12):2340-8.

26. Peytchev A, Ridenhour J, Krotki K. Differences between RDD telephone and ABS mail survey design: coverage, unit nonresponse, and measurement error. J Health Commun. 2010;15(sup 3):117-34.

27. Korkeila K, Suominen S, Ahvenainen J, Ojanlatva A, Rautava P, Helenius H, Koskenvuo M. Non-response and related factors in a nation-wide health survey. Eur J Epidemiol. 2001;17(11):991-9.

28. Littman AJ, Boyko EJ, Jacobson IG, Horton J, Gackstetter GD, Smith B, Smith TC. Assessing nonresponse bias at follow-up in a large prospective cohort of relatively young and mobile military service members. BMC Med Res Methodol. 2010;10(1):99.

29. Colsher PL, Wallace RB. Data quality and age: health and psychobehavioral correlates of item nonresponse and inconsistent responses. J Gerontol. 1989;44(2):P45-52.

30. Volken T. Second-stage non-response in the Swiss health survey: determinants and bias in outcomes. BMC Public Health. 2013;13(1):167.

31. Little RJ. Regression with missing X's: a review. J Am Stat Assoc. 1992; 87(420):1227-37.

32. Van der Heijden GJ, Donders ART, Stijnen T, Moons KG. Imputation of missing values is superior to complete case analysis and the missingindicator method in multivariable diagnostic research: a clinical example. J Clin Epidemiol. 2006;59(10):1102-9.

33. Gómez-Carracedo MP, Andrade JM, López-Mahía P, Muniategui S, Prada D. A practical comparison of single and multiple imputation methods to handle complex missing data in air quality datasets. Chemom Intell Lab Syst. 2014;134:23-33

34. Parr CL, Hjartåker A, Scheel I, Lund E, Laake P, Veierød MB. Comparing methods for handling missing values in food-frequency questionnaires and proposing $k$ nearest neighbours imputation: effects on dietary intake in the Norwegian women and Cancer study (NOWAC). Public Health Nutr. 2008; 11(4):361-70.

35. Barzi F, Woodward M, Marfisi RM, Tognoni G, Marchioli R. \& GISSIPrevenzione investigators. Analysis of the benefits of a Mediterranean diet in the GISSI-Prevenzione study: a case study in imputation of missing values from repeated measurements. Eur J Epidemiol. 2006;21(1):15-24.

36. Fraser GE, Yan R, Butler TL, Jaceldo-Siegl K, Beeson WL, Chan J. Missing data in a long food frequency questionnaire: are imputed zeroes correct? Epidemiology (Cambridge, Mass). 2009;20(2):289.

\section{Publisher's Note}

Springer Nature remains neutral with regard to jurisdictional claims in published maps and institutional affiliations.

Ready to submit your research? Choose BMC and benefit from:

- fast, convenient online submission

- thorough peer review by experienced researchers in your field

- rapid publication on acceptance

- support for research data, including large and complex data types

- gold Open Access which fosters wider collaboration and increased citations

- maximum visibility for your research: over $100 \mathrm{M}$ website views per year

At BMC, research is always in progress.

Learn more biomedcentral.com/submissions 